\title{
Jnhalt $\mathbf{I}$.
}

\section{(Sebidite find burd) * bezeidnet. \\ A. Anorbnutg nad bem $\mathfrak{3}$ halt.}

Nr.

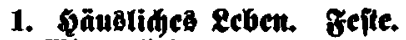

Gette

*31. $\mathfrak{G}$ of f $m a n n$, miegenlied .......... 33

*53. פ⿺ ö r i $e$, Mausfallenfprüdlein ......... 50

*54. " unjer Friß .............. 50

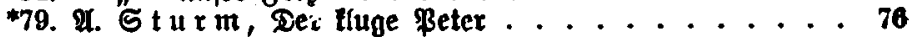

*97. Spinnerlied . . . . . . . . . . . . . 90

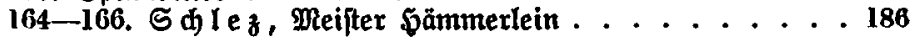

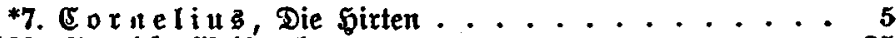

*23. (Bs r e if , Meilnnadten ........... 25

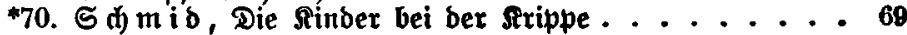

*85. Tro i a , Gin frohes Feft.......... 81

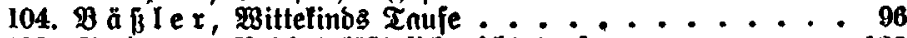

119. Bs $\mathrm{x}$ i m m, \$abbot läpt fid nidjt taufen. . . . . . 128

*30. S e $\mathfrak{y}$, Meuiahr . . . . . . . . . . . 32

4. Dieffenbad, Der Dfterhas ........ 8



110. $\mathfrak{B}$ o c e l $\mathfrak{l}$, Der Erntelranz . . . . . . . . . 111

*59. $\mathfrak{R}$ e i ll i

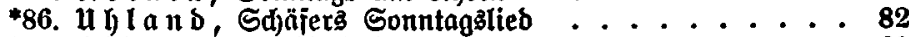

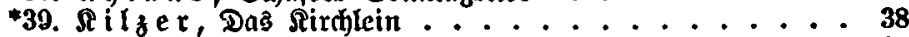

*69. Sh ille r, Bum Jeburtatag .........67

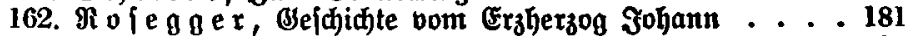

*94. Bugelljudjeit .............. 98

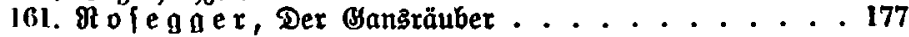

\section{Meisheit. Sottoertrauen.}

*80. S u terme ifter, Sdulze soppe madt bas 28 etter . . 77

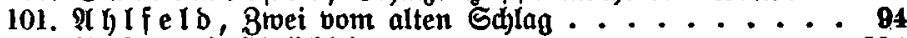

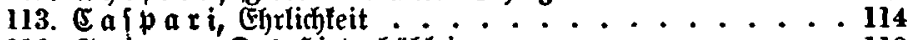

116. ङ $\mathrm{r} \mathrm{im} \mathrm{m}, \mathfrak{D a s}$ Sirtenbüblein . . . . . . . 118

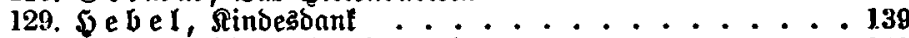

130. " Einer ober ber andere ........140

132. " Der \$err Stabtridter ......... 143

159. Ne ilu i d, Der Pfennig. . . . . . . . . 173

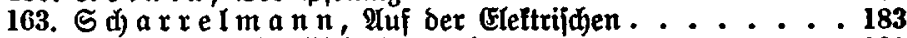

171. T r o i a n, Die Bidłtelmännđen ......... 199

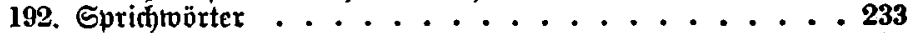

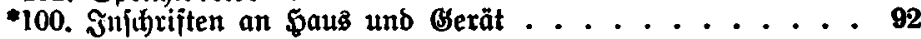

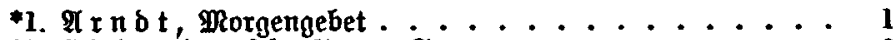

*. Eideno orff, Sotteg Segen .......... 


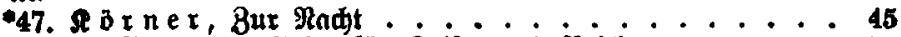

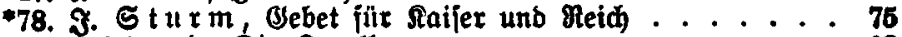

*87. $\mathfrak{u}$ G $I$ a $\mathrm{b}$, Die Sapelle ........... 82

"86. "Edäjers Eonntagstieb ........ 82

160. R e in $i d$, Der Dieb ................... 175

\section{2eutinge Befüinte anb Cage.}

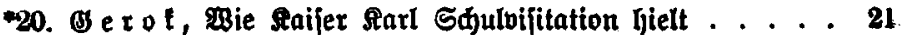

-21. "Wie Raifer Rarl ßareiben lernte ....... 22

*93. $\mathfrak{B}$ o"g 1 , Seinrid ber Boglex ........... 87

"67. Sallet, Der Derfflinger . . . . ....... 65

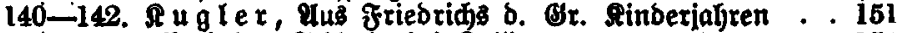

143. Tad ber Sdjladt bei Rorlin ........ 154

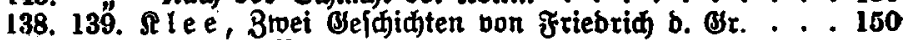

*6. E 0 I $\mathbf{h}$ or $\mathfrak{n}$, Nemteremtemtem ......... 5

102. $\mathcal{A} \mathrm{r}$ (d) e $\mathrm{n} \mathfrak{l}$ o $\mathfrak{l}$, Der gejangene $\mathfrak{D u}\{a \mathrm{r} . . . . . .94$

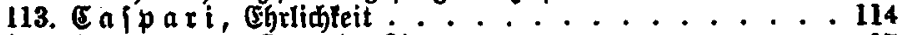

17. F o nt a ne, Der alte Bieten .......... 17

*68. Ga a l et, Bieten ............ 66

-18. Fout a $\mathrm{f}_{\mathrm{e}}$, Sendrib............. 18

*15. ff $\mathfrak{i}\{$ di $\mathrm{e} r$, Der alte Frib auf Sanßjpuci . . . . . . 14

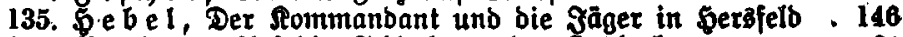

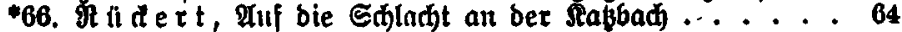

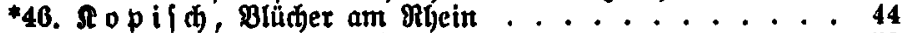

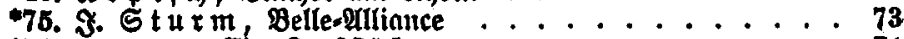

76. Cin ภunt thä ..........74

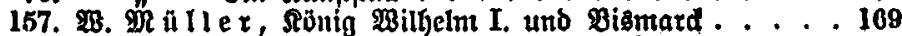

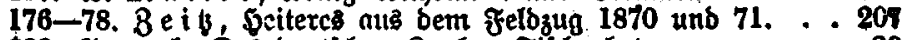

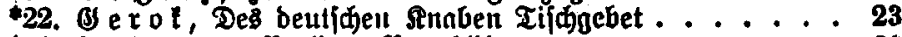

19. ₹̊ o t tane, Berliner Republifaner ........20

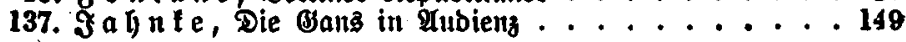

193. Die Nibelungen . . . . . . . . . . . . . 235

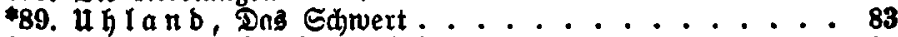

"90. "Eiegfrieds Edwert ........ 84

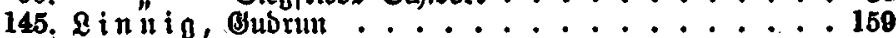

146. 162

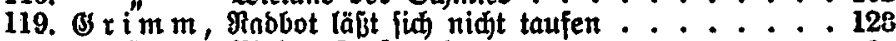

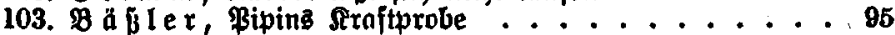

104. . . . . . . . OB

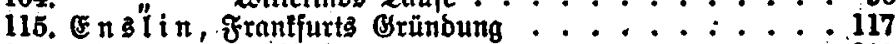

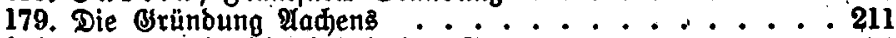

73. Simr o ḋ, Die Shule ber Stuber........72

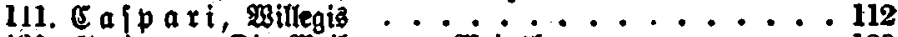

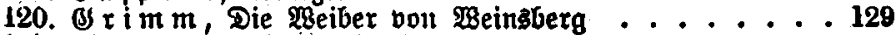

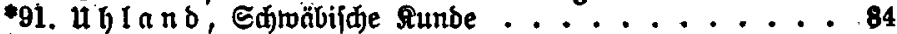

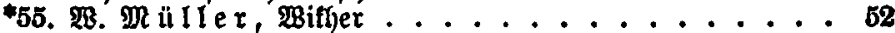

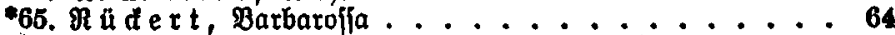

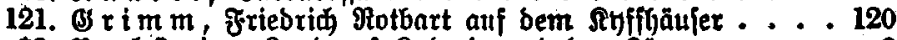

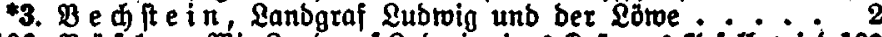



*92. $\mathfrak{u}$ h lan $b$, Graf cberłarbs 98 eißbom ....... 86 
Rr. Seise

180. Det Ebetftein in ber brandenturgifiben srone . . . . 212

112. Ea $\{$ p a $\mathbf{i}$, Die Sulfiten vor Naumburg . . . . . . 118

72. Sel bel, Die Zräume ........... . . 70

181. Der Zobeśtwürfel ................ 213

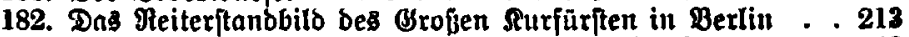



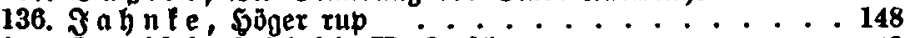

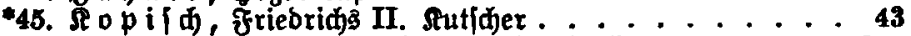

183. Das Sauts mit ben Sđafştöpen in Berlin ...... . 214

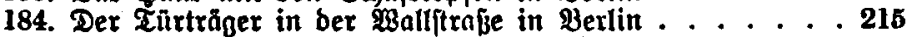

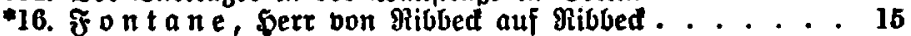

185. Die Faullheitâprobe zu Tübingen ........... 21t:

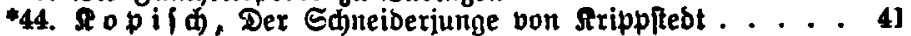

43. 4 Die Broerge auf bem Baum........ 40

\section{Eagen bet Griedsen unb vomer.}

194. Die Oötter ber Brieđjen . . . . . . . . . . . 251

195. Serfules. . . . . . . . . . . . . . . 257

106. Däbalus unb ğaruş . . . . . . . . . . . . . 264

107. Plus ber Sage bon Troja . . . . . . . . . . . 207

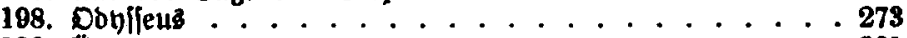

109. Altuend . . . . . . . . . . . . . . 291

200. Die Brünbung গtoms . . . . . . . . 203

\section{2cutfáce Ranb und Bolt.}

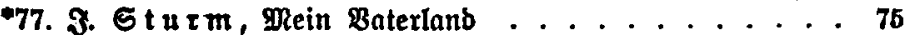

109. $\mathrm{B}$ o del, Die alte Linbe........... 109

110. . Der Erntefranz ........ I11

186. Cin "Epaziergang unter ben Linben in Berliı. . . . 216

187. Frnulfurt am פhain .............. 219

188. Illcinfałyrt bon Mainz bis söln . . . . . . . . 221



\section{2as fibrige Curopa.}

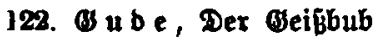

\section{Erembe Erbteile.}

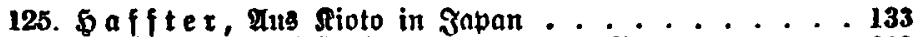

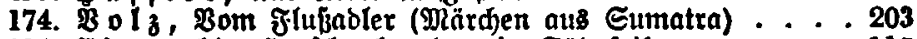

114. Ei in wa Ib, Jeufhredenplage in Silbajrifa . . . . 115

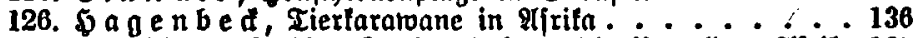

151. 152. Ml a n s fe l b, Bwei Dlärđjen a. b. Urivalb v. Xffrila 165

189. Erfter Sdulanfang in Bamum .........223

190. Lagerleben in Deutjh-Ditafrifa .......... 227

\section{Rierleben unb pflamenleben.}

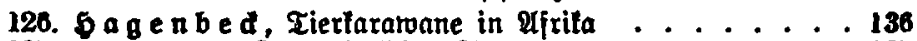

127. Der gebulbige Sóre........ 137

128. Gebădnis ber Raubtiere . . . . 188 
ax.

*3. Bedfein, Lanbgraf Lubwig unb ber Löwe . . . . . 2

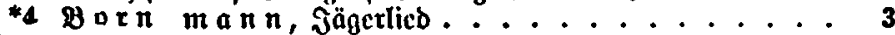

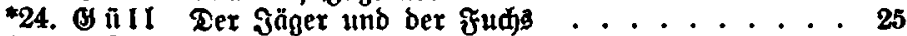

*56. $\mathfrak{B}$ f e f f el, Die zruei Đunbe ............. 63

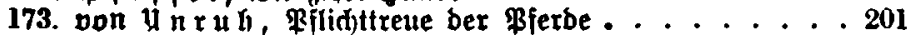

*84. $\mathfrak{T}$ o i a $n$, \$ajenleben................ 81

*06. Tie beiben sajen ................... . 90



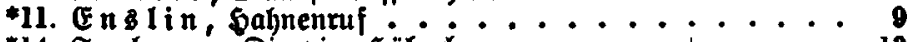

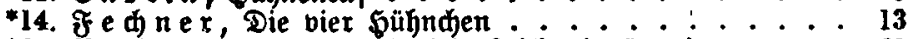

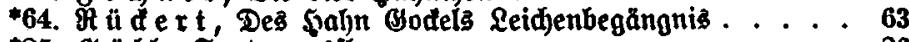

*25. \& if II, હpatyenauaflutg . . . . . . . . . . . . 26

*54. M ó r i f e, Unjer Frib. . . . . . . . . . . . . . 50

\%05. Der befte Bogel. . . . . . . . . . . . . . . . 89

*94. 2ogelbođzeit .................... 88

167. Se i bel, Sas Golbhähndjen . . . . . . . . . . 189

168. $\operatorname{Der}$ Baunlönig ............. 101

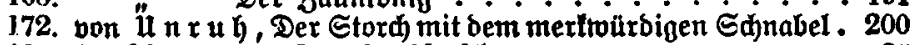

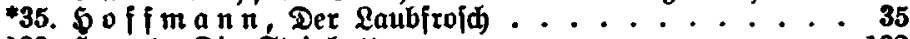

123. $\mathfrak{\downarrow}$ a a $\mathbf{s}$, Die Eteinbutte .............. 132

124. "Der Stör................. 133

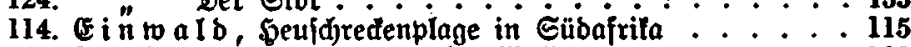

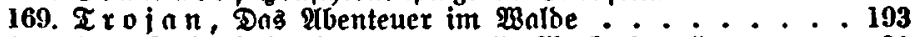

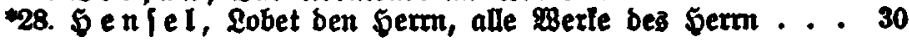

*41. S I e t le, Der Îpfellaum . . . . . . . . 40

*16. F o nt a ne, Gerr von Ribbed ......... 15

109. $\mathfrak{B}$ o de $\mathrm{l}$, Die alte Linbe........... 109

110.

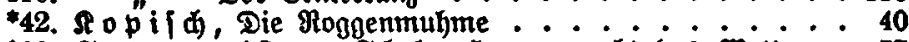

*80. Euterme $i$ ft er, Edjulze \$oppe madt bas Better. 77

*57. $\Re$ e in if , Frïhlingshloten $\ldots \ldots \ldots$

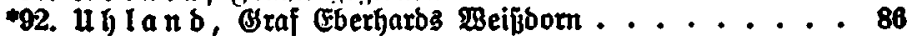

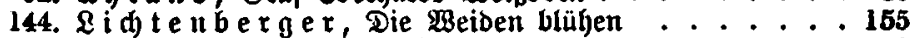

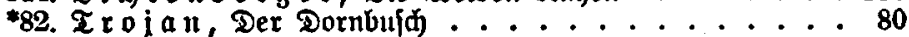

170. Linien-unfraut ............. 197

*74. 5 tö́ e

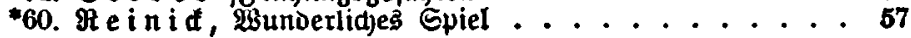



\section{.8. Zeben ber Grbe. Jahresgeiten. Zagegzeitem.}

155. M e i is ner, Sonne und 2 Binb ......... 168

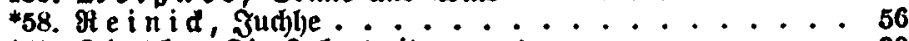

*40. I letle, Die Jahreģzeiten . : . . . . . . . 39

*12. F a lle, Die Eorgliden ........... 11

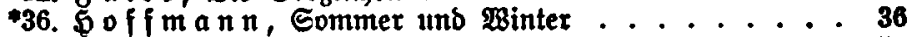

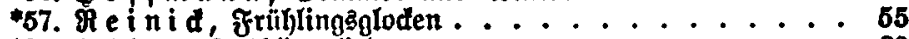

*38, $\mathfrak{s}$ o $\mathfrak{l} \mathfrak{t} \mathfrak{y}, \mathfrak{f}$ rühlingslied ............ 38

*32-34. $\mathfrak{p}$ of f $m$ a $n \mathrm{n}$, frriublingalieder ........ 33

*74. St o b e $r$, frühlingaggefahren ......... 73

*5i. \& o we nftein, April ............47

61.

3uni

48

gellel, gejebud 8.13 Iuf.

M. 20 
Pe.

*48. I r u m m a d e r, Sonntagglied im Sommer . . . . . 48

*61. $\mathfrak{A}$ e i n i d, Der Serbf . . . . . . . . . . . . . . 58

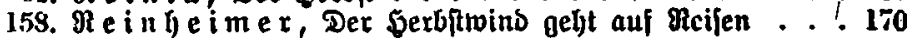

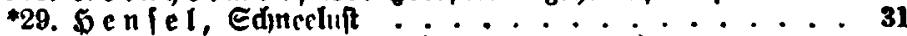

*10. En 8 I in, Edjlitfduhlauf . . . . . . . . . . . . g

*37. $\mathfrak{T}$ o f f $\mathrm{m}$ a $n$, Der Cislauf . . . . . . . . . . . 37

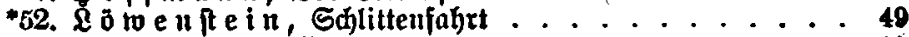

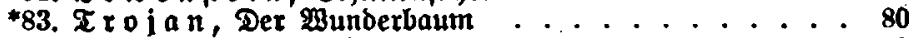

*1. $\{x \mathfrak{n} \delta \mathrm{t}$, Morgengebet ................. I

*0. Eidendorff, (Sottes Eegen . . . . . . . . . . . 9

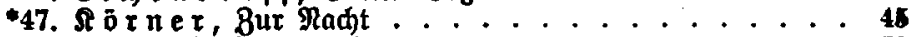

71. S e i o el, Eaternenlied. . . . . . . . . . . . . 70

\section{B. Inorbum! nad ber Form. \\ Lehrlyaftes (Dibaftifdies).}



147-150. \&u th e $r, \mathfrak{B i e r} \mathfrak{F a h e l n ~ . . . . . . . . . . . ~} 163$

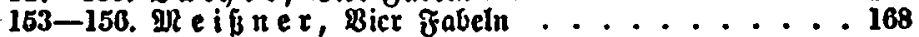

*56. B feffel, Die zlvei Sunde ..........53

102. Epriduwerter . . . . . . . . . . . 233

100. Julfhriften auf \$aus und OSerät . . . . . . . . 02

426, of îll, Rätjcl ............... 27

*49. \& z we n ftein, nätjel ...........47

*81. Su t c $r$ m e $i$ ft e $r$, Redfragen unb Rntworten . . . . 77

*08. 09. Năttel ................ 01

191. Nätfel . . . . . . . . . . . . 231

\section{(Erzählenbes (Epifhes).}

\section{Märdjer.}

*63. Ir a å e $r t$, Der Spielmann ............61

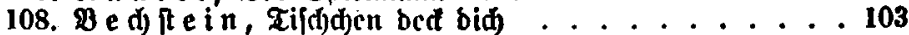

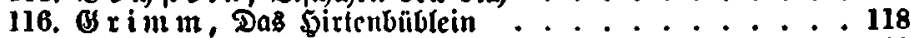

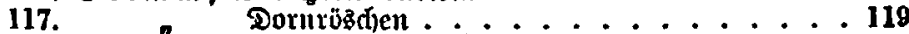

118. "Der Frieder und bas Satjerlieşdjen . . . 123

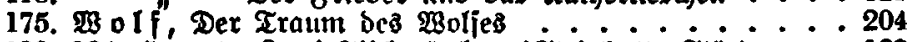

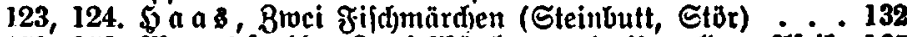

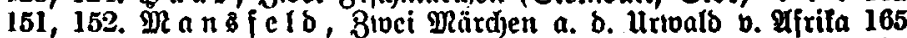

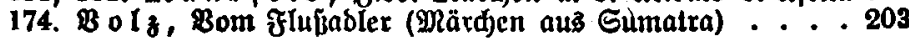

19. Eage. Regenbe.

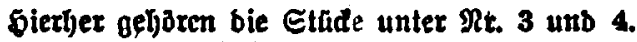

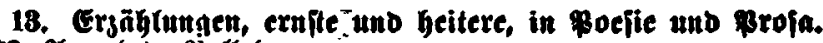

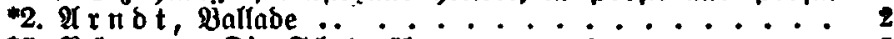

*5. $\mathfrak{B} \mathfrak{a} \mathfrak{r} \mathfrak{g}$ er Die Edjabgräber .

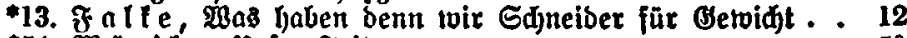

"54. Mör i le, unfer frik. ............ 50

*80. Sut er m c ifter, Sdjulze \$oppe madt bas æaetter . . 77

101. U h If el b, Bloci bom alten Edjlag. .......94

113. E a \{p a r $i$, Ebrlid)teit ............ 114 
ST.

129-135. Se bel, Erzăhlıtngen . . . . . . . . . . 139

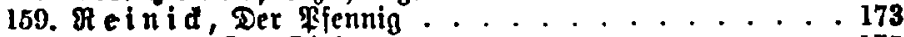

160.

101. No feg ger, Der Ganšräuber......... 177

162.

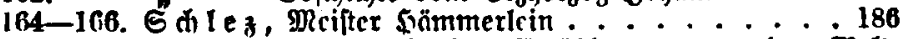

Gierber geborren aud biele ber Frẳilungen aus ber Weltgejajichte unb viele Eagen ( $9 x .3$ mi 4).

\section{Eniberutg und sciforcibung.}

109. $2 B$ b d $I$, Die alte Sinbe.

110. Der Erntefranz. . . . . . . . . . . 111

122. G u be, Der Beißbub . . . . 130

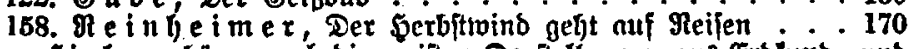
Gierher gehören aud) bie meiften Daritellungen aus (Exblunbe unb Raturlunde (Nr. 5 bis 0 ). Afor

\section{Sefable unb Bebanten (\&nrifbeb).}

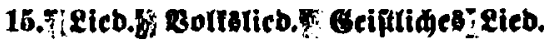

4. Bornem a nn, Jăgerlieb . . . . . . . . . 3

*7. Eornelius, Die Sirten............ . 7

27. 5 a u f f, Mciters Mornengefang . . . . . . . . . 29

-28. Se n je 1, Lobet ben \$errn ........... 30

*31-37. Đo f $m$ a $n \mathfrak{n}$, finberlieber ........ 33

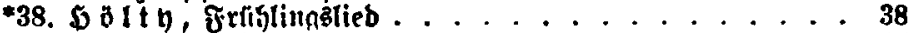

*47. \& 8 rner, Bur Radt .............45

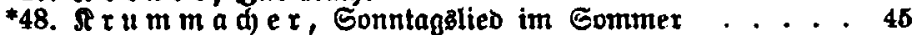

*71. Se i b e l, gaternenlieb. . . . . . . . . . 70

*77. $\mathfrak{G} \mathbf{t} \mathbf{r} \mathrm{m}$, Mein Baterland ........... 75

*78.

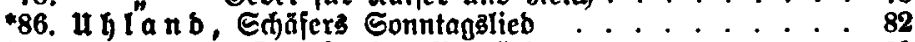

*88.

*07. Spinnerlieb................ . 90

\section{Sanblung (Dramatif̧eş).}

\section{Ceipräd). Eecbitgeiprāa.}

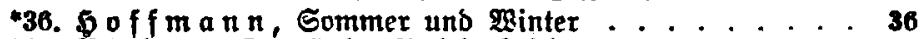

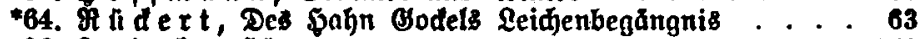



144. Li b te nb erger, Die Beiben blühen. . . . . . 168

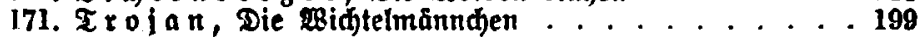

*14. f e d) ner, Die vier \übnden . . . . . . . . . . . . 13

*25. of î Ir, Evakenansflug. . . . . . . . . . . . . . . 20

*61. 8 o to enftein, Juni ............ 48

*53. פr or ite, Maugjallenipriddlein .......... 50

*60. Re in id, פunberlides Epiel. . . . . . . . . . . . 67

61.

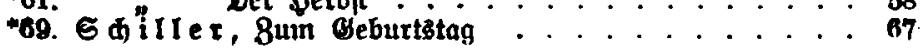

